\title{
Vehicle Localization Based on Visual Lane Marking and Topological Map Matching
}

\author{
Rabbia Asghar $^{1}$, Mario Garzón ${ }^{2}$, Jérôme Lussereau ${ }^{1}$, Christian Laugier ${ }^{1}$
}

\begin{abstract}
Accurate and reliable localization is crucial to autonomous vehicle navigation and driver assistance systems. This paper presents a novel approach for online vehicle localization in a digital map. Two distinct map matching algorithms are proposed: i) Iterative Closest Point (ICP) based lane level map matching is performed with visual lane tracker and grid map ii) decision-rule based approach is used to perform topological map matching. Results of both the map matching algorithms are fused together with GPS and dead reckoning using Extended Kalman Filter to estimate vehicle's pose relative to the map. The proposed approach has been validated on real life conditions on an equipped vehicle. Detailed analysis of the experimental results show improved localization using the two aforementioned map matching algorithms.
\end{abstract}

Index Terms-Map Relative Localization, Topological Map Matching, Lane Level Matching, Autonomous Vehicles.

\section{INTRODUCTION}

Localization is one of the key components of the system architecture of autonomous driving and Advanced Driver Assistance Systems (ADAS). Accurate localization is crucial to reliable vehicle navigation and acts as a prerequisite for the planning and control of autonomous vehicles. An indepth literature overview of various localization techniques is presented in [1].

Offline digital maps are readily available especially in urban scenarios and they play an important role in field of autonomous vehicles and ADAS. Modern navigation approaches use offline maps not only for route planning but also for tactical decision making. Moreover, for situational awareness, localization of the vehicle within the map is very important. It is common in the literature to fuse different types of sensors to improve the vehicle's localization. Fusing an offline map with on-board vehicle sensors provides complementary benefits. An accurate map helps integrate acquired sensor data and also provides information that is outside of sensor's reach. In this work, we use an offline map database to define grid and topological maps and assist online localization of the vehicle.

We propose a novel approach that fuses GPS and dead reckoning sensors along with two map matching algorithms to implement online pose estimation of the vehicle. Due to their affordability and convenience, Global Navigation Satellite System (GNSS) receivers together with dead reckoning sensors are commonly used for localization of the vehicle. However, this conventional methodology is not sufficient

\footnotetext{
1 Univ. Grenoble Alpes, Inria, 38000 Grenoble, France, email: FirstName.LastName@inria.fr

${ }^{2}$ Univ. Grenoble Alpes, Inria, Grenoble INP, 38000 Grenoble France, email: mario.garzon-oviedo@inria.fr
}

to achieve accurate localization[2]. Additional to GPS and dead reckoning, our work brings together both lane level and topology of the map and fuses the information using Extended Kalman Filter (EKF) to improve map relative localization.

Our strategy is based on using similar features, as those used for navigation of the vehicle for reliable localization. For instance, an autonomous vehicle needs to be aware of the lane markings on the road to effectively maintain the lane keeping of the vehicle. We use lane tracker to correct the vehicle's pose by aligning visual lane markings to lanelevel grid map. Similarly, it's a common practice to use Topological Map Matching (TMM) for route planning. We use TMM as another observation in EKF to correct the vehicle position.

The main contribution of this paper is a novel approach that incorporates both visual lane-level map matching and TMM with EKF. Instead of independently implementing TMM with GPS alone, we integrate it with the multisensor fusion EKF. We perform online topological map matching using the already fused information from GPS, dead reckoning and ICP based lane matching. This reduces the error variance of the estimated vehicle position and consequently, improves map matching. While the lane-level map matching is the primary source of map relative observation, the topological map matching also contributes to the EKF by making observation to the filter in case pose estimate drifts off the road.

The paper is organized as follows. Section II discusses related work to map relative localization. Section III describes the localization architecture with an in-depth explanation of the two map matching techniques. Section IV presents experimental results and analysis. Finally conclusions are drawn in section $\mathrm{V}$.

\section{RELATED WORK}

Both online and offline map matching algorithms have been extensively explored in past three decades [3]. Accordingly, there has been a growing trend for incorporating map matching into localization of the vehicle, by using the map itself as an additional information.

Among features based localization, lane level map matching approaches are most popular. With the advancement in computer vision and machine learning based methods, cameras are widely used for the extraction of lanes and other visual features. Pink et al. uses visual lane markings as features to match with lane level map generated from aerial imagery. It uses ICP for matching with no additional sensors 
[4]. Another work uses dead reckoning to keep a history of lane markings defined as a registry, instead of just using the short ranged visual lane markings in front of the ego vehicle, and matches this registry with the map [5]. It uses non probabilistic filter to fuse map matching results with dead reckoning. Apart from cameras, laser scanners are also used for features based localization, such as lane markings [6], crossroad detection [7] and landmarks [8].

Many works have used EKF to fuse map matching information with other sensors. Tao et al. proposed to use EKFbased algorithm for fusing GPS, lane markings and dead reckoning without using grid maps. A video camera system is used to detect and parameterize lane marking and estimate the lateral distance and heading information to the vehicle [9]. Gruyer et al. presents a similar EKF based approach that uses two lateral cameras to detect ego-lane markings, with emphasis on improving lateral map relative localization [10]. However, in contrast with our work, the topology of the road network is not taken into account in the previous two approaches. Apart from EKF, particle filters are popularly used to localize the vehicle on lane or road network ([11], [12], [13]), since particle filters allow for multi-hypothesis map matching in case of ambiguous situations.

Topological map matching approaches are used in the scenarios where the vehicle always travels on road. Drevelle et al. takes into account the topology of the map and solves the localization by defining it as a constraint satisfying problem [14]. Another approach proposes a particle filter based map matching algorithm that uses camera lane marking to compute likelihood of the matched road [15]. Their proposed map matching algorithm also respects the topology of the lane-level map. Similar to our work, Najjar et al. uses EKF for map relative localization. It proposes to use belief theory to find the probability of the map matched road segments and select the most likely segment. Their approach however does not take visual features in account [16]. A grid based method is proposed in Matthaei et al. [17], where lane markings or building lines on the grid can be defined using both camera and laser scans. They propose to use topological map matching with GPS, map and extracted features to make an initial guess of the pose and further fuse the map matched pose with odometry and inertial sensors with help of an EKF.

To summarize, the main difference in our work and previous ones is that we perform TMM as well as lane level matching using the already fused information from GPS and dead reckoning. Moreover, TMM observations to EKF are non-periodic and are only made if the vehicle's estimated pose drifts out of its lane. We show with real sensor data experiments that our proposed method can improve robustness and accuracy of the map relative localization.

\section{MAP RELATIVE LOCALIZATION}

\section{A. System overview}

The map relative localization approach described here is based on Extended Kalman filtering, as illustrated in Fig. 1. The multisensor EKF fuses GPS and dead reckoning comprising of INS feedback and wheel odometry, as well as two distinct map matching algorithms. The state vector $X_{k}$ consists of vehicle's pose, twist and linear acceleration in three-dimensional space. Vehicle's kinematic model is defined by classical unicycle model. We assume the reader is familiar with fusion of INS and odometry in an EKF for a unicycle model and thus only the map matching algorithms and their fusion with EKF is discussed in detail.

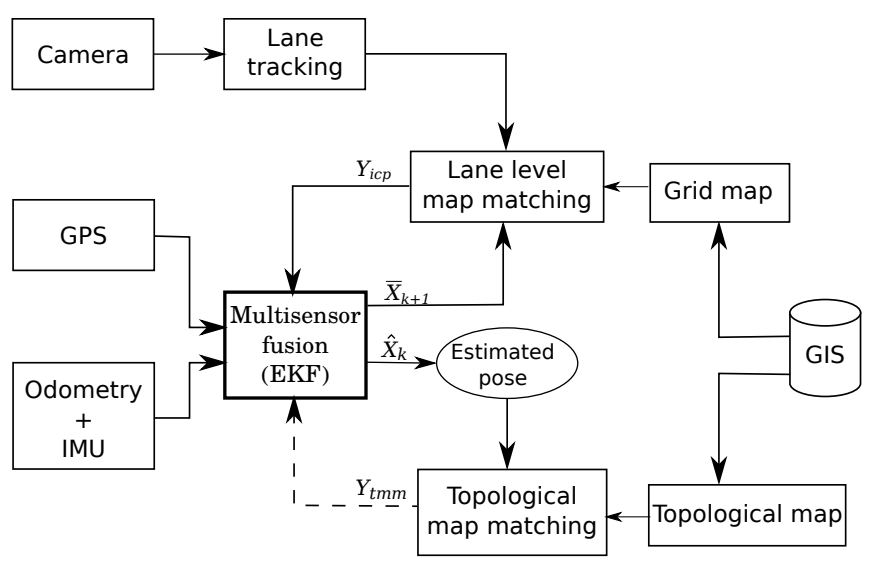

Fig. 1: Overview of the map relative localization approach.

Geographical Information System (GIS) in Fig. 1 represents offline map database that provides layouts of the drivable lanes and central lane markings. Both lanes and central lane markings are comprised of segments of varying lengths. A lane segment is defined by two points in WGS84 coordinates along with lane width and the direction of road traffic.

To incorporate GPS measurements and map matching in local navigation frame, a local tangent plane is defined using East, North, Up (ENU) coordinate frame with its origin defined at arbitrarily chosen fixed coordinates. This is acceptable for a small map within a radius of few kilometers with the assumption that the change in earth curvature is negligible. By convention $x$-axis of the frame points to the East, $y$-axis to the North and $z$-axis is oriented upwards with respect to WGS84 ellipsoid. The pose of the vehicle in $2 \mathrm{D}$ is defined by vehicle's position $(x, y)$ and it's heading $\theta$.

GPS measurements are converted to ENU coordinates and the position of the vehicle in $2 \mathrm{D}$ is used for measurement update. GPS observation is only made when the vehicle is in motion and GPS error is used to define it's covariance matrix.

The details of lane markings and topological map matching based localization as shown in Fig. 1 are discussed in the sections III-B and III-C respectively.

\section{B. Lane-level map matching}

For lane marking based localization, we translate the map information to a 2D grid representation, hereby referred to as map grid. WGS84 coordinates are converted to a local ENU coordinates system. The map grid illustrated in Fig. $2 \mathrm{a}$, has a resolution of $10 \mathrm{~cm}$ and represents 3 spaces: road in white, central lane marking in gray and no road in black. With an accurate and reliable localization, this same map 


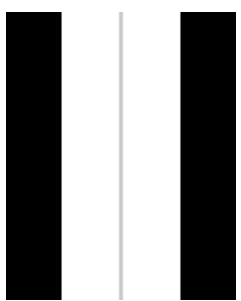

(a)

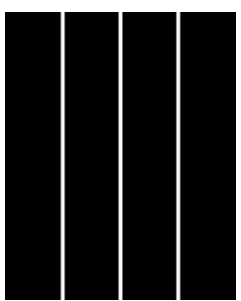

(b)

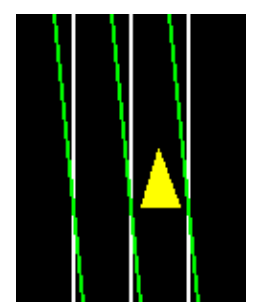

(c)
Fig. 2: Lane level map matching on grid map with lane tracker. a) original map grid, b) extracted lane markings in white, c) lane tracker based lane markings projected in green with respect to ego vehicle's pose (yellow triangle).

grid can be potentially fused with occupancy grid [18] and aid in situational awareness and decision making.

We extract the central lane marking and road edges, signifying the side lane markings, to perform lane-level map matching. They are illustrated by white cells in Fig. $2 \mathrm{~b}$ and represent target points for map matching algorithm. A particle filter based lane tracker [19] is used, that takes camera image and camera's intrinsic and extrinsic parameters as input and provides road parameters i.e. road width, curvature of the road, vehicle's lateral displacement with respect to ego lane marking. This information along with the EKF predicted pose of the ego-vehicle $\bar{X}_{k+1}$ is used to project potential lane markings points on the map grid. These points are illustrated by green cells in Fig. 2c and represent source points. They are projected upto $10 \mathrm{~m}$ ahead of the vehicle with steps of $0.25 \mathrm{~m}$. For each source point, corresponding lane marking target points (cell matches) on the map grid are selected using nearest neighbor search. 2D Iterative Closest Point (ICP) algorithm [20] is used to make pose correction between the sets of source and corresponding target lane marking points. EKF predicted pose $\bar{X}_{k+1}$ is taken as the initial guess for ICP. The corrected 2D pose that aligns source points to target lane marking points on grid map is introduced as a new observation for EKF. ICP observation equation is defined in Eq. 1.

$$
Y_{i c p}=\left[\begin{array}{l}
x_{i c p} \\
y_{i c p} \\
\theta_{i c p}
\end{array}\right]=\left[\begin{array}{lll}
1 & 0 & 0 \\
0 & 1 & 0 \\
0 & 0 & 1
\end{array}\right]\left[\begin{array}{l}
x \\
y \\
\theta
\end{array}\right]+m_{k}
$$

where $x_{i c p}, y_{i c p}$ and $\theta_{i c p}$ represent ICP corrected 2D pose of the vehicle in ENU coordinate frame and $m_{k}$ represents the observation error. Assuming that $m_{k}$ in the ICP observation is Gaussian distributed and the noise sources of position and orientation are independent, error covariance matrix $Q_{i c p}$ is given in Eq. (2).

$$
Q_{i c p}=\left[\begin{array}{ccc}
\sigma_{x}^{2} & \sigma_{x y}^{2} & 0 \\
\sigma_{x y}^{2} & \sigma_{y}^{2} & 0 \\
0 & 0 & \sigma_{\theta}^{2}
\end{array}\right]
$$

While the observation is in the world ENU frame, it is more intuitive to define the longitudinal $\sigma_{l o n g}$ and lateral $\sigma_{l a t}$ standard deviations in the vehicle frame. The translational components of the $Q_{i c p}$ can then be computed as follows:

$$
\begin{aligned}
\sigma_{x}^{2} & =\sigma_{\text {lat }}^{2} \cos ^{2}(\theta)+\sigma_{\text {long }}^{2} \sin ^{2}(\theta) \\
\sigma_{y}^{2} & =\sigma_{\text {lat }}^{2} \sin ^{2}(\theta)+\sigma_{\text {long }}^{2} \cos ^{2}(\theta) \\
\sigma_{x y}^{2} & =\left(\sigma_{\text {lat }}^{2}-\sigma_{\text {long }}^{2}\right) \cos (\theta) \sin (\theta)
\end{aligned}
$$

$\sigma_{\theta}$ represents standard deviation of vehicle's heading. Detailed derivation of the covariance matrix can be referred to in Najjar et al. [16].

Fault detection scenarios are used to verify if the ICP corrected pose is suitable for EKF observation. Error metric in ICP is defined by sum of squared distances between corresponding points. If the metric error does not fall below a threshold in a given number of iterations, the ICP is considered non convergent. For experiments, this threshold was set to $0.6 \mathrm{~m}^{2}$ for every respective pair of source and target points. In some cases, it is possible that the ICP is convergent but corrected pose is still incorrect, e.g. in case of convergence to local minima. To take this into account, a separate threshold is set for acceptable translational and heading correction, based on the assumption that the vehicle is heading parallel to the direction of road. The experimental values used for this translational and heading threshold were $10 \mathrm{~m}$ and $10^{\circ}$ respectively. In case, the ICP is non convergent or the threshold is crossed, ICP observation for the EKF is skipped.

\section{Topological map matching}

Our map database contains lane-level waypoints which for the sake of TMM are defined as a topological graph comprising of nodes (points defined in WGS84 coordinates) and directional edges, identified by pairs of nodes. The map matching algorithm uses if-then decision-rule approach [21] to snap the vehicle on to the most appropriate edge. Initially, the algorithm defines a search area of fixed radius around the estimated vehicle position. For experiments, this radius was set to $30 \mathrm{~m}$. All the edges partially or completely part of this radius and oriented within $90^{\circ}$ of the vehicle's heading are shortlisted. This way for any road, only the lanes feasible for the respected vehicle direction are considered. This is illustrated in Fig. 3. For the ego-vehicle positions in green, only the edges in blue are considered for TMM.

With availability of new vehicle pose estimate, TMM algorithm snaps on to the closest edge to vehicle's position. Along the stretch of snapped edge $E_{n}$, the algorithm orthogonally projects the vehicle's position on the edge and selects it as the snapped point. The snapped point represents topologically map matched position $P_{t m m}$ as represented by a cross in Fig. 3 for respective ego-vehicle pose. For every new edge snapped $E_{s}$, the algorithm verifies that the current snapped edge $E_{n}$, has a feasible path to the new edge. If not, TMM algorithm enters a conflict resolution mode. This conflict may occur near an intersection or two separate roads running close by. In such a scenario, it is unknown whether $E_{n}$ or $E_{s}$ are incorrect (Fig. 3 illustrates a TMM conflict scenario where $E_{n}$ is incorrectly identified). Thus, TMM algorithm awaits a subsequent edge $E_{s+1}$ to correct the map matching and resolve the conflict. Subsequent edge is not 


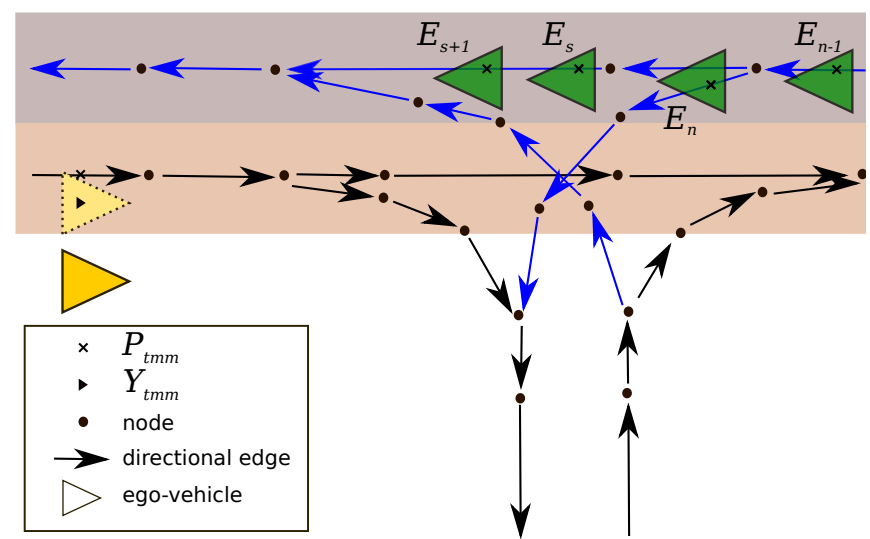

Fig. 3: Topological map at a 3-way intersection. Nodes and edges are defined on the center of lane respectively. Green triangles illustrate position of the ego-vehicle at four instants representing a TMM conflict scenario; TMM relevant edges are shown in blue. Yellow triangle represents a different case where the ego-vehicle's estimated position is outside of lane, and the position shown by dotted triangle is the TMM observation.

necessarily the next edge in the map. In straight sections of road, an edge can be of significant length. If the vehicle moves some distance from the conflict position, the same edge can be subsequent edge. We set this distance to $4 \mathrm{~m}$ signifying the vehicle has moved at least its own length.

The conflict resolution is illustrated in the flow diagram in Fig. 4. The algorithm checks if any of the previous two edges, $E_{n-1}$ or $E_{n-2}$, has a feasible path to the new edge $E_{s}$ and then to the subsequent edge $E_{s+1}$, this holds true for conflict shown in Fig. 3. Otherwise, if that is not the case, alternate new edge, Alt $E_{s}$ from shortlisted ones is considered. Candidate alternate edges are part of the search areas and they are considered one by one in order of increasing proximity. Should no feasible path be found with alternate edges as well, the TMM conflict is not resolved. The TMM is stopped and re-initialized with an warning.

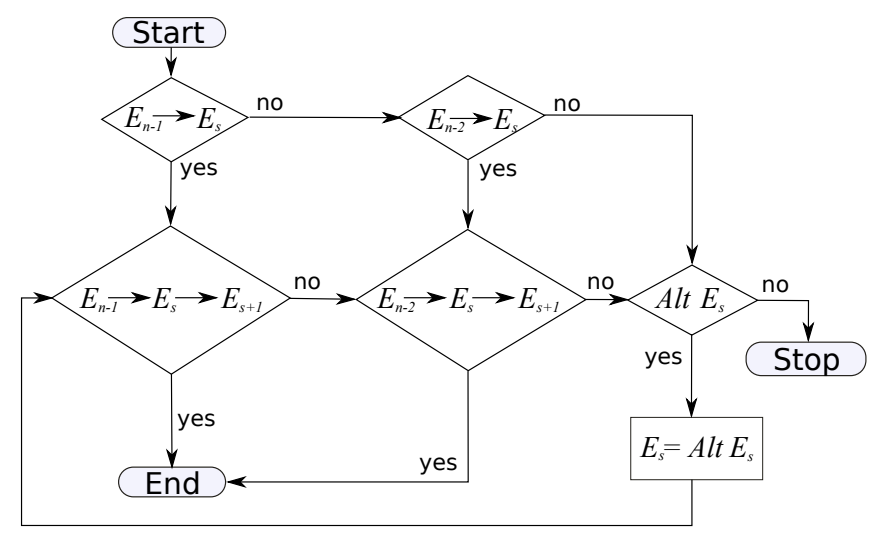

Fig. 4: Flow diagram of conflict resolution in TMM.

Since TMM algorithm operates by snapping on to an edge, it always provides information about vehicle's posi- tion $\left(P_{t m m}\right)$ strictly on the center of the lane. However, the vehicle's actual position can be anywhere along the width of the lane. In consequence, making a periodic TMM observation as a Gaussian distribution with mean position value $P_{t m m}$ will be inappropriate. Therefore, we propose to only make the EKF observation when the estimated pose of the vehicle is out of lane. To represent this, TMM observation to EKF is shown by a dashed line in Fig. 1. An example scenario is illustrated in Fig. 3 where yellow ego-vehicle's estimated position is outside of lane. TMM observation $Y_{t m m}$ is determined by computing point $\left(x_{t m m}\right.$, $\left.y_{t m m}\right)$ on the lane section of map matched edge that is closest to estimated vehicle's position. This point is represented by the black triangle in pale yellow ego-vehicle. The TMM observation equation (Eq. 3) is defined in the ENU coordinate frame, similar to ICP observation Eq. 1. $n_{k}$ represents the TMM observation error.

$$
Y_{t m m}=\left[\begin{array}{l}
x_{t m m} \\
y_{t m m}
\end{array}\right]=\left[\begin{array}{ll}
1 & 0 \\
0 & 1
\end{array}\right]\left[\begin{array}{l}
x \\
y
\end{array}\right]+n_{k}
$$

The covariance matrix $Q_{t m m}$ is defined in the same manner as ICP covariance matrix $Q_{i c p}$, except with only position components. The observation for EKF is skipped if the TMM algorithm is in a conflict mode.

\section{VALIDATION METHODOLOGY}

\section{A. Experimental setup}

Experiments were conducted on a Renault Zoe vehicle, shown in Fig. 5. For the proposed localization approach described earlier, low-cost GPS, IMU, vehicle's wheel odometry and a front camera were used.

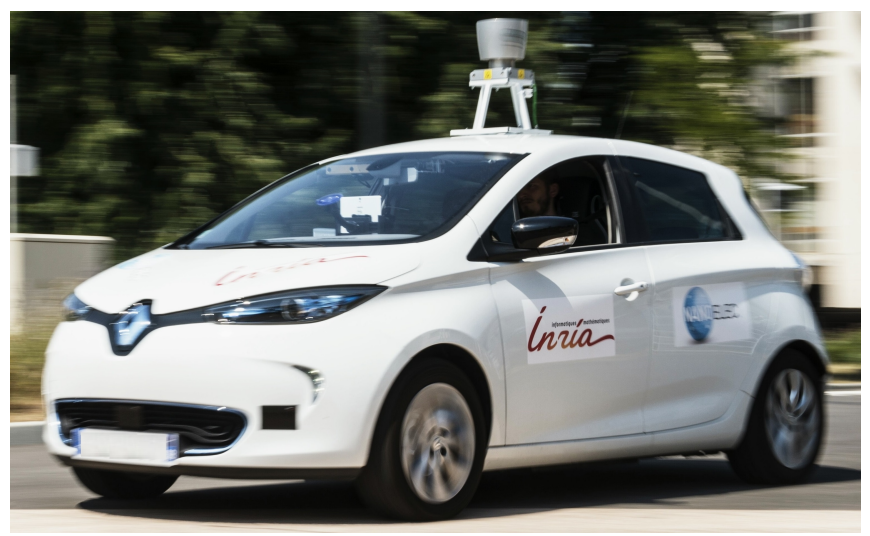

Fig. 5: Experimental platform.

Additionally OpenStreetMap, shown in Fig. 6, was used to extract the relevant latitude and longitude information needed to define grid and the topological map. The test course used for experiments is about $2.2 \mathrm{~km}$ long and includes a speed bump and a roundabout. The curved sections of the road are not continuous curves and have discrete representation in the map. However, we assume the OSM map to be accurate. The experiments were performed with varying weather conditions from sunny, cloudy to drizzling. 
The software is written in Python and $\mathrm{C}++$ inside the ROS framework. The grid map is defined such that it is compatible with ROS map_server. The update rate for GPS, wheel odometry, IMU measurements and EKF are $2 \mathrm{~Hz}$, $50 \mathrm{~Hz}, 100 \mathrm{~Hz}$ and $100 \mathrm{~Hz}$ respectively. The lane level map matching algorithm updates at $10 \mathrm{~Hz}$. TMM observations are non-periodic. During the tests, the vehicle runs at an average speed of $60 \mathrm{~km} / \mathrm{h}$. Lane changing or overtaking scenarios are not considered.

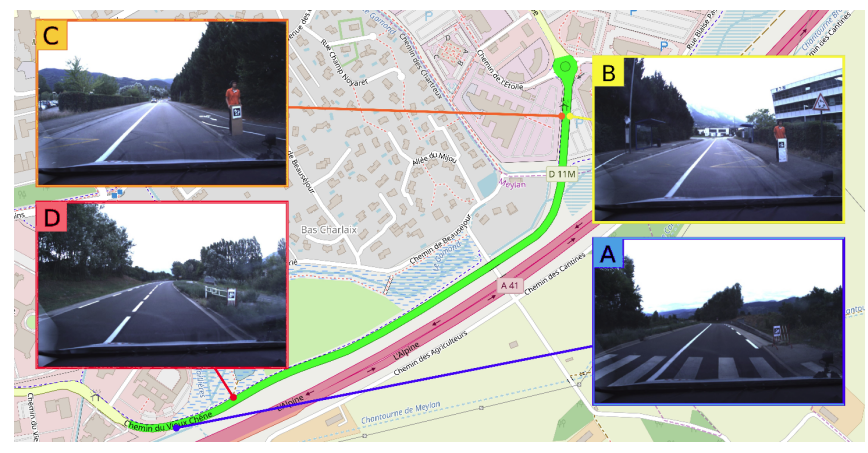

Fig. 6: Snapshot of OpenStreetMap illustrating the test route and respective positions of AprilTag markers.

We use AprilTag visual fiducial detection algorithm [22] at 4 fixed and known positions in the map in order to evaluate the performance of localization algorithm. Fig. 6 illustrates the respective positions of the tags and experimental route on OpenStreetMap. Each tag faces the direction of traffic of the right side lane. Therefore, vehicle can capture only one tag in any region. The AprilTag algorithm provides transformations of a tag with respect to vehicle. We use this information along with the tag's position in map to find error in estimated position of the vehicle. Markers A and D are placed on the start and end of the traversed route, both of them are at curved sections of the road. Markers B and C are placed before and after the roundabout respectively.

\section{B. Comparison of localization approaches}

Three sensor fusion use cases are considered to compare and characterize our proposed approach.

1) GPS and dead-reckoning (DR) only

2) GPS, DR and lane-level map matching

3) GPS, DR, lane-level and topological map matching

As a measure of deviation in the localization results in each of these three approaches, we take note of the time interval during which the estimated vehicle position remains within its lane. Map relative localization in or out of lane is a qualitative measure of lateral displacement on straight sections of road and also longitudinal displacement on curved sections.

Table I summarizes the results as percentage of the total run time for a test distance of $70 \mathrm{~km}$. Since there is no map matching involved in the GPS and dead-reckoning only approach, the vehicle in this case moves in and out of the lane sporadically and the estimated pose was noted to be within the lane for even less than $50 \%$ of the time. Often,

\begin{tabular}{|c|c|c|c|}
\cline { 2 - 4 } \multicolumn{1}{c|}{} & \multicolumn{3}{c|}{ Estimated pose remains within lane } \\
\hline Approach & GPS+DR & $\begin{array}{c}\text { GPS+DR+ } \\
\text { Lane-level MM }\end{array}$ & $\begin{array}{c}\text { GPS+DR+ } \\
\text { Lane-level MM+ } \\
\text { Topological MM }\end{array}$ \\
\hline mean & $43 \%$ & $97 \%$ & $99 \%$ \\
\hline st. deviation & $9 \%$ & $0.7 \%$ & $0.3 \%$ \\
\hline median & $44 \%$ & $97 \%$ & $99 \%$ \\
\hline min & $18 \%$ & $95 \%$ & $98 \%$ \\
\hline
\end{tabular}

TABLE I: Comparison of localization approaches based on percentage of total runtime during which estimated vehicle pose remained within the lane. Our proposed approach with lane-level and topological map matching outperforms others.

the main source of error in the localization of vehicle being the GPS error itself.

With introduction of the proposed lane level map matching, the algorithm is efficiently able to localize the vehicle within the lane for a mean of $97 \%$ of the runtime. In our approach, the TMM observation is briefly introduced only when the vehicle pose has already drifted out of the lane. In the last column of Table I, it is apparent that TMM improves robustness of localization by further reducing the time interval in which the vehicle's estimated pose drifts out of the lane. Figure 7 shows a curved section of the road, where the estimated pose of the vehicle drifts out of lane in all three approaches. One can observe the red path with our proposed approach has fastest recovery towards the lane. The blue path without TMM takes longer time, the green path illustrating the GPS and DR only approach widely goes out of the lane and recovers slowest.

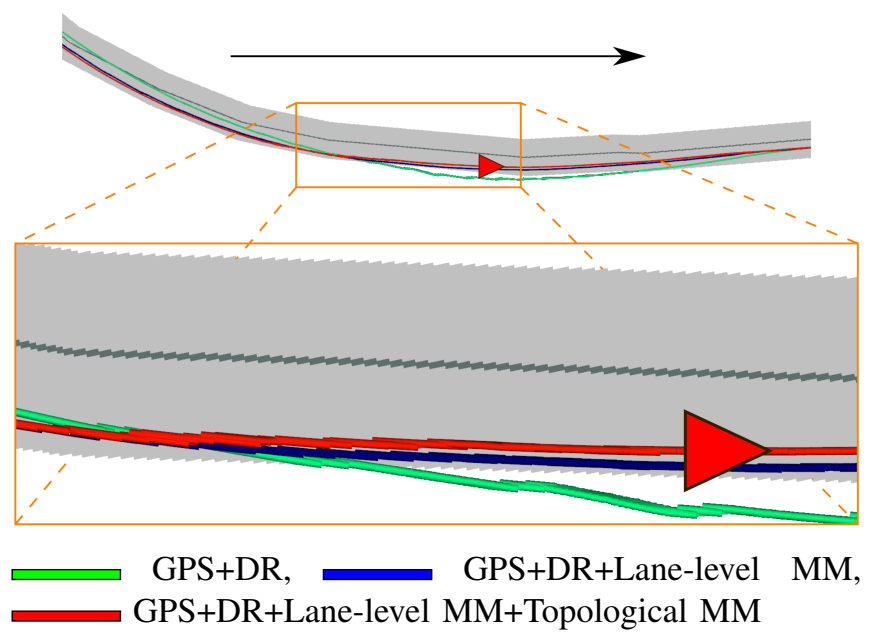

Fig. 7: Estimated pose of the vehicle using three different localization approaches on a curved section of road. It is visible that our proposed approach with TMM recovers fastest when the pose drifts out of lane. The vehicle is provided as a reference where the estimated pose is still partially out of lane. Black arrow represents direction of travel.

Furthermore, in our experiments, TMM assures proper localization of the vehicle where lane level map matching fails, for instance at the roundabout, which results in lane 
level map matching getting rejected. However, the proposed algorithm works as intended during and after the roundabout.

\section{Results}

Apart from qualitative evaluation, we have validated the proposed localization approach with respect to 4 fixed positions, previously explained in IV-A. For each tag frame detected, lateral and longitudinal error in position of the vehicle was determined using vehicle's heading. Fig. 8 and Table II show the statistics of lateral and longitudinal position errors for each tag for a total run of $35 \mathrm{~km}$.

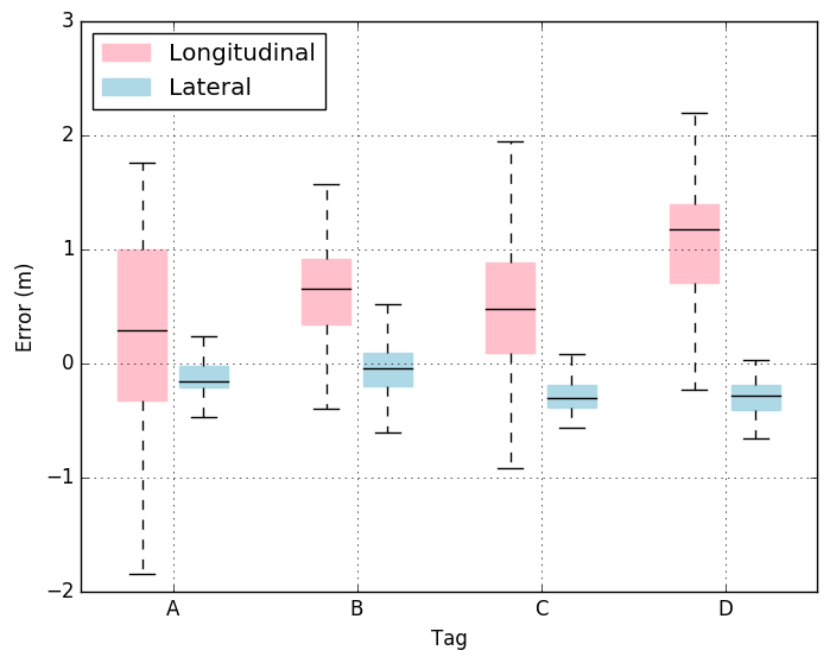

Fig. 8: Box plot of longitudinal and lateral position errors at 4 respective tags using the proposed localization approach for total test runs of $35 \mathrm{~km}$.

\begin{tabular}{|c|c|c|c|c|}
\hline \multirow{2}{*}{ Tag } & \multicolumn{2}{|c|}{ Longitudinal error (m) } & \multicolumn{2}{c|}{ Lateral error (m) } \\
\cline { 2 - 5 } & mean & st. deviation & mean & st. deviation \\
\hline A & 0.81 & 0.60 & 0.17 & 0.11 \\
\hline B & 0.67 & 0.38 & 0.22 & 0.20 \\
\hline C & 0.61 & 0.46 & 0.29 & 0.13 \\
\hline D & 1.17 & 0.51 & 0.29 & 0.16 \\
\hline
\end{tabular}

TABLE II: Statistical values for absolute longitudinal and lateral position error at 4 respective tags.

The lateral errors for all tags remained within $1 \mathrm{~m}$ with a mean absolute value of $0.25 \mathrm{~m}$. This also conforms with the observation that estimated vehicle position always remained within the lane in the test area close to tags. All tags have maximum longitudinal error within $2.5 \mathrm{~m}$, with an absolute mean value of $0.8 \mathrm{~m}$. Tag A was placed near the start of test run, where the initial estimated position was every time at an offset. Therefore, longitudinal localization error at Tag A varied significantly at every run. Moreover, both Tags $\mathrm{A}$ and $\mathrm{D}$ were recognized at region of high vehicle speed, $50 \mathrm{~km} /$ hand $60 \mathrm{~km} / \mathrm{h}$, which also contributed to larger localization error.

\section{CONCLUSION AND FUTURE WORK}

This paper has presented a new approach to map relative localization that fuses visual lane level and topological map matching with GPS and dead reckoning. Both matching algorithms described are based on common features that are used in assistance for the navigation of the vehicle. The proposed method has been validated using readily available, open source map. Three use cases were studied, to compare different levels of sensor fusion: with both the map matching algorithms, only lane level map matching algorithm and neither of them. It was shown that two algorithms together can robustly localize the vehicle within the lane. Finally, accuracy of the localization of the vehicle was established at four points of interest using visual tags.

Future research will study the use of RTK GPS to define a high definition map with well-defined curved sections of the road and more detailed lane markings. That will further increase the accuracy and robustness of the localisation approach. We also plan to integrate lane change assist to support localization in scenarios where the vehicle overtakes or changes lane. Furthermore, we will use the estimated pose of the vehicle to fuse the grid map with ego-centric occupancy grid to contain information whether a respective cell is free or occupied and whether it is part of the road and navigable.

\section{ACKNOWLEDGMENT}

These researches have been conducted within the ANRT FUI TORNADO project. The authors also wish to thank Agostino Martinelli for his fruitful input and discussions and Jean-Alix David for his help with experiments.

\section{REFERENCES}

[1] S. Kuutti, S. Fallah, K. Katsaros, M. Dianati, F. Mccullough, and A. Mouzakitis, "A survey of the state-of-the-art localization techniques and their potentials for autonomous vehicle applications," IEEE Internet of Things Journal, vol. 5, no. 2, pp. 829-846, 2018.

[2] F. Zhang, H. Stähle, G. Chen, C. C. C. Simon, C. Buckl, and A. Knoll, "A sensor fusion approach for localization with cumulative error elimination," in 2012 IEEE International Conference on Multisensor Fusion and Integration for Intelligent Systems (MFI). IEEE, 2012, pp. 1-6.

[3] M. Hashemi and H. A. Karimi, "A critical review of real-time mapmatching algorithms: Current issues and future directions," Computers, Environment and Urban Systems, vol. 48, pp. 153-165, 2014.

[4] O. Pink, "Visual map matching and localization using a global feature map," in 2008 IEEE Computer Society Conference on Computer Vision and Pattern Recognition Workshops. IEEE, 2008, pp. 1-7.

[5] R. P. D. Vivacqua, M. Bertozzi, P. Cerri, F. N. Martins, and R. F. Vassallo, "Self-localization based on visual lane marking maps: An accurate low-cost approach for autonomous driving," IEEE Transactions on Intelligent Transportation Systems, vol. 19, no. 2, pp. 582-597, 2017.

[6] J. Levinson, M. Montemerlo, and S. Thrun, "Map-based precision vehicle localization in urban environments." in Robotics: Science and Systems, vol. 4. Citeseer, 2007, p. 1.

[7] A. Mueller, M. Himmelsbach, T. Luettel, F. v. Hundelshausen, and H.J. Wuensche, "Gis-based topological robot localization through lidar crossroad detection," in 2011 14th International IEEE Conference on Intelligent Transportation Systems (ITSC). IEEE, 2011, pp. 20012008.

[8] T. Weiss, N. Kaempchen, and K. Dietmayer, "Precise ego-localization in urban areas using laserscanner and high accuracy feature maps," in IEEE Proceedings. Intelligent Vehicles Symposium, 2005. IEEE, 2005, pp. 284-289.

[9] Z. Tao, P. Bonnifait, V. Fremont, and J. Ibanez-Guzman, "Mapping and localization using gps, lane markings and proprioceptive sensors," in 2013 IEEE/RSJ International Conference on Intelligent Robots and Systems. IEEE, 2013, pp. 406-412. 
[10] D. Gruyer, R. Belaroussi, and M. Revilloud, "Map-aided localization with lateral perception," in 2014 IEEE Intelligent Vehicles Symposium Proceedings. IEEE, 2014, pp. 674-680.

[11] I. Miller, M. Campbell, and D. Huttenlocher, "Map-aided localization in sparse global positioning system environments using vision and particle filtering," Journal of Field Robotics, vol. 28, no. 5, pp. 619643, 2011.

[12] W. Lu, E. Seignez, F. S. A. Rodriguez, and R. Reynaud, "Lane marking based vehicle localization using particle filter and multikernel estimation," in 2014 13th International Conference on Control Automation Robotics \& Vision (ICARCV). IEEE, 2014, pp. 601-606.

[13] I. Szottka, "Particle filtering for lane-level map-matching at road bifurcations," in 16th International IEEE Conference on Intelligent Transportation Systems (ITSC 2013). IEEE, 2013, pp. 154-159.

[14] V. Drevelle and P. Bonnifait, "igps: Global positioning in urban canyons with road surface maps," IEEE Intelligent Transportation Systems Magazine, vol. 4, no. 3, pp. 6-18, 2012.

[15] F. Li, P. Bonnifait, and J. Ibañez-Guzmán, "Map-aided dead-reckoning with lane-level maps and integrity monitoring," IEEE Transactions on Intelligent Vehicles, vol. 3, no. 1, pp. 81-91, 2018.

[16] M. E. El Najjar and P. Bonnifait, "A road-matching method for precise vehicle localization using belief theory and kalman filtering," Autonomous Robots, vol. 19, no. 2, pp. 173-191, 2005.

[17] R. Matthaei, G. Bagschik, and M. Maurer, "Map-relative localization in lane-level maps for adas and autonomous driving," in 2014 IEEE Intelligent Vehicles Symposium Proceedings. IEEE, 2014, pp. 49-55.

[18] L. Rummelhard, A. Nègre, and C. Laugier, "Conditional monte carlo dense occupancy tracker," in 2015 IEEE 18th International Conference on Intelligent Transportation Systems. IEEE, 2015, pp. 2485-2490.

[19] J. Lussereau, P. Stein, J.-A. David, L. Rummelhard, A. Negre, C. Laugier, N. Vignard, and G. Othmezouri, "Integration of adas algorithm in a vehicle prototype," in 2015 IEEE International Workshop on Advanced Robotics and its Social Impacts, 2015.

[20] S. Rusinkiewicz and M. Levoy, "Efficient variants of the icp algorithm." in 3dim, vol. 1, 2001, pp. 145-152.

[21] C. A. Blazquez, J. Ries, and P. A. Miranda, "Towards a parameter tuning approach for a map-matching algorithm," in 2017 IEEE International Conference on Vehicular Electronics and Safety (ICVES). IEEE, 2017, pp. 85-90.

[22] J. Wang and E. Olson, "AprilTag 2: Efficient and robust fiducial detection," in 2016 IEEE/RSJ International Conference on Intelligent Robots and Systems (IROS). IEEE, oct 2016, pp. 4193-4198. 